Voix et Images

volxetimages

\title{
Sauver la pensée. Entretien avec Suzanne Jacob
}

\section{Lori Saint-Martin et Christl Verduyn}

Volume 21, numéro 2 (62), hiver 1996

Suzanne Jacob

URI : https://id.erudit.org/iderudit/201233ar

DOI : https://doi.org/10.7202/201233ar

Aller au sommaire du numéro

Éditeur(s)

Université du Québec à Montréal

ISSN

0318-9201 (imprimé)

1705-933X (numérique)

Découvrir la revue

Citer ce document

Saint-Martin, L. \& Verduyn, C. (1996). Sauver la pensée. Entretien avec Suzanne Jacob. Voix et Images, 21(2), 224-233. https://doi.org/10.7202/201233ar d'utilisation que vous pouvez consulter en ligne.

https://apropos.erudit.org/fr/usagers/politique-dutilisation/ 


\title{
Sauver la pensée Entretien avec Suzanne Jacob
}

\author{
Lori Saint-Martin, Université du Québec à Montréal \\ et Christl Verduyn, Université Trent
}

V. et I.: Vous avez commencé par faire de la chanson, avant même d'écrire de la fiction?

S. J.: J'ai fait un peu de théâtre, d'abord au collège, puis au Théâtre de la Boulangerie en 1964, avec les Apprentis-Sorciers. La chanson est venue ensuite. Mais le premier roman, Flore Cocon, paraitra en 1978, avant le premier disque. L'écriture a commencé au collège vers l'âge de quatorze ou quinze ans, à travers le journal étudiant, mais surtout par une correspondance presque quotidienne pendant toute cette période du collège, qui a duré quatre ans. Mais je n'ai jamais appelé ça de l'écriture.

V. et I. : Voyiez-vous des parentés entre l'écriture de chansons et l'écriture de fiction?

S. J.: Ce sont deux écritures complètement différentes pour moi. J'écris une chanson pour ma voix et avec ma voix, et pour la scène. Ce n'est pas du tout le même matériau que pour le roman. Par contre, je peux dire que c'est grâce à la chanson si j'ai pu organiser un budget qui m'a donné le temps d'écrire.

V. et I. : Quelle a été votre formation? A-t-elle alimenté votre écriture?

S. J.: J'ai fait ce qu'on appelait à l'époque le baccalauréat ès arts. J'ai été pensionnaire à Nicolet pendant six ans, dans un petit collège entouré d'une école de théâtre, d'une école de musique et d'une école de peinture. C'était la maison mère des Sœurs de l'Assomption, une communauté religieuse québécoise. Le collège était dirigé par une femme intelligente qui désirait nous former non pas à l'obéissance mais au discernement. Les auteurs à l'Index ou les films frappés par la censure, elle croyait que nous devions en prendre connaissance, que ça faisait partie de notre formation que d'examiner ce que notre société interdisait et de trouver pourquoi. Cette passion du discernement a certainement joué un grand rôle dans tout ce qui s'est passé pour moi ensuite.

V. et I. : C'était donc un milieu exceptionnel? 
S. J.: C'était un milieu animé par quelques femmes réveillées et passionnées. Je n'ai compríis que beaucoup plus tard qu'une occasion exceptionnelle n'est souvent saisie que par les gens prêts à la saisir. Ceux qui sont déjà endormis ne vont rien saisir du tout et ça n'est pas...

V. et I. : C'est déjà trop tard.

S. J.: Oui.

V.et I.: Lisiez-vous beaucoup alors? Y a-t-il des écrivains qui vous ont marquée?

S. J.: C'était un lieu où on lisait tout le temps, la musique, le théâtre, le cinéma, les livres, tout. Vers 1962, 1963, je me suis rendu compte qu'on allait sortir du collège sans avoir étudié le nouveau roman. J'ai demandé qu'on le mette au programme. Eh bien, une prof est allée suivre un cours d'été sur le nouveau roman à Laval, et elle nous l'a donné en septembre. Assez formidable, non? Bon, mais oui, un écrivain m'a profondément marquée pendant cette période. Je pense depuis longtemps que c'est lui et lui seul qui m'a amenée à ce qu'on convient d'appeler aujourd'hui l'écriture. Il s'agit de Pierre Jean Jouve, celui qui intègre dans le roman la vitesse de la poésie, comme au cueur de la mort, la vie. Dès l'ouverture d'Hécate, on comprend que la prose vient de s'affranchir du devoir de la preuve ou de l'illustration "probante". Autrement dit, la prose se délivre de sa volonté lourde et dérisoire d'y pouvoir quelque chose et nourrit sa liberté au cour même des paradoxes.

V. et I. : Et quels sont les auteurs que vous lisez aujourd'hui?

S. J.: Ces dernières années, il y a eu Thomas Bernhard, Peter Handke. Récemment, j'ai découvert Marlen Haushofer, une autre auteure autrichienne. Il y a quelque chose qui se passe du côté de. l'Autriche. La première femme à faire une thèse sur mes livres, c'est une Autrichienne. Marlen Haushofer, c'est une écriture complètement organique, cohérente, tout le temps juste dans le souffle; dans le chant. C'est ce qui m'intéresse: des écritures qui ne sont pas monstrueuses, qui sont dans une harmonie ...

V. et I. : Que voulez-vous dire par une écriture "monstrueuse"?

S. J.: C'est une voix qui ne s'appartient pas, où il y a des morceaux qui sont collés, ajoutés, des bras à quelqu'un, des mains à quelqu'un d'autre, des yeux empruntés à on ne sait plus qui.

V. et I. : Une écriture inauthentique, donc?

S. J.: La monstruosité peut être parfaitement sincère... Enfin, je pense qu'il y a des monstres authentiques.

V. et I.: On remarque, dans votre ceuvre, toute l'importance que prend l'individu par opposition au collectif. Cette importance de l'individu soulève la question du rapport à deux mouvements sociopolitiques, le féminisme et le nationalisme, la souveraineté du Québec. Commençons par le 
féminisme. "On trouve dans vos livres une critique très sévère de l'image de la femme dans la société. Vous donnez à vos protagonistes le droit de transgresser, d'oser un acte délinquant, et c'est une sorte de permission de s'assumer comme les femmes qu'elles voudraient être au lieu de se plier aux normes sociales. Mais quand elles agissent, c'est un acte très solitaire, qui n'est pas fait en. collectivité avec diautres femmes, et qui souvent, d'ailleurs, les conduit à la mort. Des actes isolés de ce genre peuvent-ils vraiment faire une différence, changer les choses?

S. J. : Je ne crois pas que l'histoire se fait en groupe, la création non plus. Il y a des années d'apparente noirceur tótale, des années et des années de gens qui prennent des routes apparemment complètement solitaires, et ces routes s'attachent patiemment les unes aux autres, et lentement, elles convergent vers cet instant très court d'une histoire qu'on va appeler la révolution, et où toute cette lente convergence souterraine va surgir à travers un groupe qui va montrer le sens collectif de tout ce courage que vous appelez individuel. Pour moi, c'est l'acte égoïste qui s'oppose à l'acte collectif, jamais l'acte individuel. Si vous me dites que ma main vient de faire un geste individuel, vous me dites que ma main s'est séparée de mes muscles et de mes nerfs, qu'elle est devenue folle, et vous avez raison. Pourtant, on réussit à dire qu'il y a un acte individuel qui s'oppose au collectif. Pour moi, ni Laura Laur, ni Gạlatée, ni Julie de L'Obéissance, ni Flore ne sont séparées des muscles et des nerfs de la société dans laquelle elles vivent. Ni folles, ni égoïstes, ni seules non plus. Elles sont solidaires d'un savoir, d'une attache, d'une relation la plupart du temps souterraine, ou en rhizome, avec toutes les femmes et toute l'humanité et toute leur société, et ça leur coûte toute leur vie, et même leur mort... Ce n'est peut-être pas cette solidarité qui leur coûte la mort d'ailleurs...

V. et I. : Quelle en serait la cause?

S. J.: C'est comme un feu qui s'éteint. Le personnage a tout consumé. C'est à ce moment-là que quelqu'un risque à nouveau tout ce qu'on lui a donné, puis il se consume.

V. et I.: Vous mettez en scène des femmes qui habitent un espace incertain, l'espace de l'imprécision ou de l'incertitude, et qui vivent dans un état de solitude. Au début de L'Obéissance, Julie dit: "Ne pas y être, voilà le sort de la majorité." Pour poser la question de l'espace au niveau politique, au niveau de la nation, est-ce que vos personnages "y sont"? S'engagent-ils dans un projet, par exemple, un pays, ou restent-ils dans un espace individuel plutôt que collectif?

S. J: : Ce qui est sûr, c'est que c'est la langue qui les construit. La langue, c'est la matière même du collectif et la matière même des personnages et de leur histoire. Je ne vois pas la langue de mes romans comme imprécise et incertaine. Or, c'est la langue qui est l'espace de mes personnages, un 
espace éminemment collectif que le travail sur la langue ne cesse de révéler et de brasser. Et c'est la langue seule aussi qui peut rendre compte des tensions entre l'inconscient collectif et la conscience individuelle.

V. et I. : On a l'impression que vous attachez au collectif, au social, parfois, des valeurs extrêmement négatives. Quand on pense à ces voitures, à l'heure de pointe, dans lesquelles tous les chauffeurs sont immobilisés et incapables de réfléchir, il y a là une société aveugle qui va à sa perte. Vos personnages ne peuvent être qu'en rupture avec cette société-là s'ils veulent préserver la moindre trace d'une personnalité.

S. J.: Pas tant la trace d'une personnalité que la trace d'une pensée. Big Brother, c'est maintenant, c'est tout de suite, et les décibels sont là pour qu'on ne puisse plus s'en apercevoir. Tous ceux qui résistent à ce progrès qui consiste à passer deux ou trois heures de loisir quotidien à être enchaînés dans des voitures dans les hurlements de la radio sont les résistants actuels. Quand vous demandez qu'on baisse le volume de la musique dans une grande librairie parce que vous n'arrivez pas à bouquiner si on vous injecte de force dans les nerfs des écrans sonores qui vous empêchent de rencontrer les voix qui cherchent à se faire entendre dans les livres, vous n'êtes pas en train d'essayer de sauver vos petites oreilles personnelles et individuelles, vous êtes en train d'essayer de sauver toute la pensée. Et la plupart du temps, vous trouvez en face de vous un robot qui vous explique qu'il n'a pas le choix lui non plus. Selon lui, c'est Toronto, ou Washington, qui lui a dit de mettre le volume à sept et si l'inspecteur du volume survient, le robot va se faire renvoyer. Là-dedans, ce ne sont pas vos petites oreilles que vous tentez de sauver, vous n'êtes nulle part dans une lutte individualiste. C'est la pensée que vous tentez de sauver. C'est une résistance épuisante, compliquée. Parce que le fait de passer pour excentrique, ou hystérique, ou migraineuse, là où c'est la pensée qui est en jeu, est épuisant, et c'est la pensée qui est en jeu, là, tout de suite aujourd'hui. La pensée, mes pensées, vos pensées, toutes les pensées, voilà ce qui est en jeu. Il n'y a pas d'autres urgences à mes yeux.

V. et I. : Vous avez parlé tout à l'heure d'inconscient collectif. Justement, on retrouve aussi chez vous les traces d'un troisième discours contemporain, le discours psychanalytique. On en voit la marque dans le sens d'un certain enseignement que peut nous donner la psychanalyse - on pense, par exemple, à toute l'analyse que vous faites du rapport mère-fille. On peut voir aussi la marque de ce discours sur le plan de l'écriture, qui est chez vous pulsionnelle, très libre. Est-ce que la démarche de la psychanalyse, ou un certain discours psychanalytique, a eu de l'importance pour vous?

S. J.: Vous allez rire... Non, je n'ai pas fait d'analyse, non, je n'ai jamais eu de rapports avec tout ça, non, je n'ai pas lu Alice Miller, non, je n'ai pas lu. Mais c'était sans doute déjà dans Pierre Jean Jouve puisque le 
personnage de Catherine Crachat est dessiné à partir d'un document retraçant les étapes principales d'une analyse qui a eu lieu. Ce qui est nouveau - et c'est ça qui est drôle - c'est que quelqu'un vient d'arriver dans ma vie, un psychanalyste, qui a fait une lecture de la littérature québécoise, parue à l'Hexagone ${ }^{1}$, qui m'a rencontrée à travers mes livres et qui m'a expliqué que j'avais à voir avec tout ça. Je veux bien, surtout que son explication a été si convaincante qu'on s'est mariés.

V. et I. : Mais justement les marques étaient là avant, puisqui'il les a vues.

S. J. : Il vous expliquerait mieux que moi que les jésuites, au $\mathrm{XvI}^{\mathrm{e}}$ siècle, ont eu la surprise de découvrir que les Amérindiens connaissaient par cœur l'inconscient, le travail du rêve. Donc, les marques, en effet, précèdent parfois de très longtemps le regard. Mais il peut arriver que le regard ait du mal à dater certaines marques. En tout cas, je me suis toujours tenue loin de la psychanalyse.

\section{V. et I. : Pourquoi?}

S. J.: Je pense que je craignais que ça ne me prive de ma parole. L'autre serait toujours là en train de me harceler' et de me dire que c'est comme ça que ça se dit. Je serais devenue une sorte d'illustratrice de théorie. Je préfère voir le film avant de lire les critiques, c'est tout. Je craignais que l'analyse ne me coupe de mon propre regard sur le monde, sur les choses, par exemple sur la relation parent-enfant. Et puis, il y a eu une époque où tout le monde s'est mis à aller en thérapie comme à la messe, et moi, je me suis braquée. Je leur disais: "Faites bien attention de ne pas noyer tout le monde dans votre grosse vague." C'était certainement un univers ultra-consensuel, cet univers psy, et extrêmement mẹnaçant - la vieille femme de "Telles ${ }^{2}$, est rassurée lorsque la jeune femme lui promet que les psy ne pourront pas la voir - parce qu'on risquait toujours de tomber sous le coup des diagnostics. Les diagnostics tuent les oeuvres. Je l'ai expliqué dans ma "Conférence-fiction ${ }^{3}$ ". Depuis, je commence à pouvoir entendre que le plus mauvais psy est justement celui qui arrête un diagnostic. D'accord, pourquoi pas?

V. et I. : Revenons un instant' à vos personnages féminins. N'y a-t-il pas, entre eux tous, une parenté certaine? On observe chez vous une longue fidélité à un même personnage ou peut-être à un même questionnement qui se poursuit toujours à travers ce qu'on perçoit de l'extérieur comme une seule et même femme.

S. J. : Mes soeurs aînées étaient des jumelles identiques. J'ai été, je crois, la seule personne qui ne les ait jamais confondues. Leur apparence était, si

1. Patrick Cady, Quelques arpents de lecture, Montréal, l'Hexagone, 1995.

2. "Telles", Possibles, vol. IV, $\mathrm{n}^{\circ} 1$, automne 1979.

3. "Conference-fiction", Possibles, vol. XII, n ${ }^{\circ} 4$, automne 1988. 
on en croit le regard des autres, identique. Et pourtant, elles étaient à mes yeux tellement évidemment différentes que je n'arrivais pas à percevoir comment les autres arrivaient à les confondre. Il est possible qu'on puisse confondre mes personnages féminins, mais bien sûr, ce n'est pas moi qui peux confondre Julie et Marie, Galatée et Maude, ou Laura Laur. Par contre, qu'elles cherchent toutes à comprendre comment habiter l'architecture contemporaine, je veux dire leur société, sans s'y perdre corps et âme, ça, oui, c'est cette quête qui m'intéresse non seulement chez mes personnages, mais chez toutes les personnes.

V. et I. : C'est-à-dire?

S. J. : C'est-à-dire comment la pensée peut survivre, comment elle survit... Je crois que je ne peux pas cesser de répéter ça, de remettre en scène des personnages qui tentent de sauver la pensée, le sens d'être là.

V. et I. : On ne peut pas ne pas être frappé par l'importance du voyage, dans tous vos textes ou presque. Il semble être lié lui aussi à la liberté, au refus de se laisser enfermer dans des situations stables, fixes, où on se perd. Que représente le voyage pour vous?

S. J.: Il y a quelque chose qui tient du nomadisme en moi, qui est peutêtre la marque d'un double héritage, qui sait, juif et amérindien? Le nomadisme, ce n'est pas du tout l'errance. C'est un itinéraire très balisé, très ancré dans des lieux presque sacrés, en tout cas essentiels. Flore Cocon annonce ça, elle qui est poussée vers la montagne sacrée, vers le lieu qui lui proposera une autre structure que celle qu'elle connait déjà pour arriver à se penser et à penser le monde. Galatée va faire la même chose et ça va la conduire au bord de l'errance: chaque étape de son voyage contient une proposition on pourrait dire totalitaire, qui tente de lui arracher son âme. Quand finalement elle aboutit à l'hôtel, à Bruxelles, elle est au bord de l'errance, presque dans la folie. Mais elle ne sombre pas dans le vagabondage, elle décide de poser un acte qui est sa façon de marquer qu'elle reprend la route initiatique qui est la sienne: elle chante.

Maintenant, si je pense à moi comme voyageuse, je me dis que je suis un peu une nomade qui aurait été en quelque sorte dépossédée de la carte du parcours nomade, cette carte qui est inscrite dans la mémoire collective des nomades, et grâce à laquelle ils survivent. Je cherche à savoir où donc cette route, cet itinéraire inscrit en moi, a été perdue. Je le fais par le voyage.

V. et I.: Vous avez parlé tout à l'heure de certains auteurs autrichiens que vous aimez. Quels sont vos rapports avec l'institution littéraire québécoise, avec la littérature québécoise? Vous y sentez-vous bien intégrée? Où vous situez-vous par rapport aux textes, aux auteurs, de cette littérature?

S. J.: Bon, ce n'est pas mon travail de me situer, ce n'est pas à moi de le faire. Ma manière d'agir cependant, ç'a été de fonder avec l'écrivain Paul 
Paré, en 1978, une maison d'édition, Le Biocreux. J'y ai tout investi pendant trois ans. C'est là que j'ai dit ce que j'avais à dire au sujet de la littérature québécoise. On a édité Evelyn Dumas, Francine Péotti, Marie Lafleur, Solange Lévesque, Madeleine Ouellette-Michalska, Jacques Renaud... j'en oublie. Ça s'est terminé brutalement par un incendie qui a fait tout disparaître, même sa cause! C'était un 6 janvier, à moins quarante degrés, un 6 janvier sans assurances, pour tout vous dire.

V. et I. : C'était en quelle année?

S. J. : C'était en 1981, janvier 1981. Il n'y avait plus rien, puisque tout était ensemble dans le même lieu. Ce que je veux dire, c'est que pendant trois années, j'ai dit ce que je pensais des écrivains de mon pays en passant à l'action, en me consacrant à en publier quelques-uns.

V. et I. : Alors vous ne vous sentez ni intégrée ni en marge? Ou peut-être que vous ne vous posez pas la question de cette manière?

S. J.: J'essaie de me tenir à l'abri du ricanement actuel qui entoure les œuvres, qui affaiblit et stérilise, à l'abri du militantisme qui découpe les ceuvres pour s'en faire des slogans. L'excentricité au sens propre me convient : elle permet des mouvements que le centre, occupé à maintenir les consensus, n'imagine pas. Ça n'empêche pas certains silences d'être difficiles à comprendre, en particulier celui qui continue d'entourer L'Obéissance. Pierre Jean Jouve parle admirablement bien des enjeux que peut représenter ce silence pour l'écrivain dans En miroir.

V. et I. : Continuons justement à parler de ce roman, L'Obéissance. C'est vrai qu'il est difficile, que le sujet est pénible, bouleversant. Il y a une rupture par rapport à ce que vous avez écrit jusque-là, dans la manière de toucher un sujet tout à fait actuel, la violence faite aux enfants, et puis aussi une rupture formelle. Qu'est-ce qui est arrivé? Pouvez-vous jeter un peu de lumière sur ce qui semble vraiment un nouveau départ?

S. J.: Tant mieux s'il y a un nouveau départ. Moi, je ne le sais pas parce que je suis toujours en train de travailler là où je ne peux pas savoir. J'ai plutôt l'impression que L'Obéissance marque la fin d'un cycle, mais si c'est la fin, eh bien oui, ça peut tout aussi bien être le début, c'est la forme du cycle qui veut ça. Plusieurs personnes m'ont dit: "Enfin, Flore Cocon est revenue dans l'écriture, dans la structure, dans l'organisation, dans la liberté de la voix." Dans La Survie, j’ai pratiqué certains tons que je vais retrouver, mieux maîtrisés, dans L'Obéissance.

V. et I. : Comment avez-vous écrit ce livre? Êtes-vous partie de l'actualité, d'un fait divers?

S. J.: Ma façon de penser mes livres, c'est chaque fois d'essayer de répondre à une question à laquelle tout le monde répond toujours des trucs tout faits qui ne m'éclairent pas. Là, je me suis demandé où, à quelle heure, comment et pourquoi on apprend tous à obéir, de quel apprentis- 
sage il s'agit, puisque l'obéissance, qui a conduit aux génocides de notre siècle, entre autres atrocités, est un apprentissage. Autrement dit, on apprend ça, participer à un génocide. Ce n'est pas rien.

V. et I. : Mais pourquoi cette question-là s'est-elle posée à ce moment précis?

S. J.: En 1972 ou 1973, j'ouvre un journal et j'aperçois ce titre écrit en majuscules: "Je voulais lui apprendre à obéir". Ça racontait un infanticide. La mère disait qu'elle avait voulu apprendre à sa fille à obéir. Ça m'a donné un choc, parce que la langue le dit tout le temps, mais je ne l'entendais pas, qu'on apprend à obéir. Encore fallait-il trouver comment cet apprentissage avait pu mener jusqu'à l'infanticide, ou jusqu'au cancer de Marie, ou jusqu'à la complicité avec les Marcos, les Duvalier, les Kim Il Song ou Ceaucescu.

V. et I. : Et vingt ans plus tard, vous avez fait le livre.

S. J. : Oui, ç'a été très long, et j'ai eu des compagnes et des compagnons de route qui ont tous tenté, chacun dans son domaine, de résoudre cette même question de l'obéissance. $Y$ a-t-il une obéissance humaine qui pourrait nous éviter d'être complices des massacres qui continuent de marquer notre siècle? Hannah Arendt, Gregory Bateson, Jean-Luc Godard, parmi quelques autres, ont guidé et soutenu ma recherche.

V. et I. : Le mot "souffrance" revient constamment dans vos œuvres. Laura Laur souffre, Flore Cocon aussi, et dans L'Obéissance la souffrance est à son comble.

S. J.: Oui, c'est vrai qu'elles refusent toutes de prendre des analgésiques! Si vous avez les images de Bosnie en tête, je me demande vraiment comment vous ne souffrez pas actuellement. Eh bien, il faut reconnaitre cette souffrance, cette tension extrême où on ne comprend pas comment on n'est pas responsable du massacre, encore hier, au Guatemala, etc. Si vous consommez cette souffrance tous les jours, elle va bien quelque part. Donc, oui, ça souffre. Il y a des milliers de gens qui ne sortent plus de chez eux tellement ils ont peur de souffrir à leur tour. Ils sont comme les parents de Marie dans L'Obéissance. Ne pas sortir de chez soi, c'est une image pour dire: ne plus pouvoir sortir de ses certitudes. Comment peut-on s'appuyer sur une certitude qui autorise la torture, c'est la question que pose L'Obéissance. Et je dis que c'est la souffrance et la peur de la souffrance qui maintiennent ces certitudes meurtrières. C'est tout un travail que d'arriver à signaler à l'autre qu'on perçoit sa souffrance, surtout à celui qui est prêt à tuer pour ne pas la reconnaitre. Une partie de mon travail consiste à ça : à faire que le chant se glisse de l'autre côté du mur et fasse entendre la compassion qui met fin à l'analgésie.

V. et I. : L'Obéissance pose aussi la question du réalisme. Une partie du roman s'intitule justement "Un fait divers" et emprunte une forme 
différente de tout ce que vous avez écrit jusque-là, qui était plus délié, plus ludique. Là on se trouve devant un récit très froid, glacial, proche des faits et des gestes concrets, rédigé à la troisième personne et même au passé simple. Quels sont donc vos rapports à ce qu'on appelle habituellement "réalisme" ou à la représentation en général?

S. J.: Une prose réaliste sur les frigos, les meubles, les cuisines, les voitures et les télés, par exemple, vous trouvez ça aujourd'hui dans les catalogues. En aucun cas, cette prose ne peut susciter la frayeur qui entoure Florence dans "Un fait divers". Quand Florence est coincée par ses meubles et qu'elle va faire son spaghetti, chaque plat devient dangereux, il va peut-être sauter à la figure de quelqu'un, et ce danger marque le passage de chaque seconde. Je ne vois pas comment une écriture réaliste pourrait produire ça. L'hyperréalisme peut-être, je me dis, oui, une sorte d'hyperréalité peut produire cette terreur.

V. et I. : Pensez à Kafka. Il y a un réalisme dans La Métamorphose, par exemple, un point de départ qui est fantaisiste et puis, à partir de là, on n'est pas dans le catalogue, on est dans l'horreur.

S. J. : Oui, on appelle ça comme ça, réalisme, mais je dis...

V. et I. : Vous, vous ne l'appelez pas comme ça.

S. J.: Moi, je raconte une histoire et je cherche ce qui m'apparaît comme le meilleur moyen de faire entendre cette histoire. Et je ne sais. pas si ça s'appelle du réalisme, parce que je ne sais pas ce que le mot vient faire dans l'histoire de Florence. Tous les moments où Florence éclate, quand elle gifle son père, quand elle détruit son chapeau de mariage dans la toilette du motel, est-ce du réalisme? Je n'ai jamais vu jouer ça, moi. Mais ça se joue dans l'histoire de Florence pour montrer l'impasse dans laquelle elle s'engage et où elle va devenir cette mère féroce. Il y a une terreur telle qui s'infiltre de partout que je ne sais pas ce dont le mot réalisme pourrait bien rendre compte ici.

V. et I. : La question de la terreur soulève fatalement celle de la mère, cette femme que vous appelez "l'origine de tout". La mère parait parfois un personnage vraiment diabolique, tout-puissant et pourtant aimé de son enfant. En même temps, elle est complètement impuissante:

S. J. : La mère joue le rôle de gardienne à la fois d'un ordre qui est nécessaire à la survie de son enfant et d'un désordre où l'enfant pourra inventer sa propre histoire. La mère est la double gardienne du passé et du futur de son enfant. Elle doit trouver un équilibre entre ces deux mouvements. Entre ces deux pôles, il y a toutes les versions de mère imaginables, de la pire, la dévoreuse, à la meilleure.

V. et I.: Vous ne nous donnez pas beaucoup d'espoir pour une mère plus épanouie... 
S. J. : Ce livre, L'Obéissance, est lui-même la mère et il constitue luimême, de son propre fait, tout l'espoir du monde. La mère possible, vivante et active en nous, est en quelque sorte prouvée par ce livre puisqu'elle rejoint en nous, au moment de la lecture, tout ce qui désire le pardon, c'est-à-dire la réparation.

V. et I. : Une dernière question: que peut-on écrire après L'Obéissance? Votre prochaine publication va être un recueil de poèmes dont vous nous donnez ici des extraits. Préparez-vous un autre roman?

S. J.: Il y a encore au moins une ou deux questions qui me hantent et j'essaie d'y répondre, oui, par le roman. Ma difficulté consiste à retrouver toute ma liberté face à l'auteure de L'Obéissance. Entre elle et moi, ça doit redevenir absolument libre. Que je puisse rester le plus près possible du souffle de ce qui veut naître aujourd'hui. Voilà, c'est ça, le sujet, l'exigence du souffle. Les poèmes des Écrits de l'eau ne sont peut-être pas autre chose que l'expression de cette exigence inaugurale de l'ouverture du monde à un souffle qui n'est pas encore nommé. Leur écriture s'étend sur une période de plus de dix ans. C'est dire qu'ils ont été les chants dans lesquels a baigné l'écriture de mes romans. 